\title{
Exonuclease III-assisted Upconversion Resonance Energy Transfer in a Wash-Free Suspension DNA Assay
}

\author{
Yinghui Chen ${ }^{\dagger,}$, Hien T.T. Duong ${ }^{\S}$, Shihui Wen ${ }^{\dagger \star}$, Chao Mi ${ }^{\dagger \star}$, Yingzhu Zhou ${ }^{\dagger,}$, Olga Shimoni ${ }^{\dagger, \ddagger}$, Stella M. \\ Valenzuela ${ }^{*} \|$, Dayong Jin ${ }^{*}+$,
}

${ }^{\dagger}$ Institute for Biomedical Materials and Devices, School of Mathematical and Physical Sciences, Faculty of Science, University of Technology, Sydney, NSW 2007, Australia

${ }^{\ddagger}$ ARC Research Hub for Integrated Device for End-user Analysis at Low-levels (IDEAL), Faculty of Science, University of Technology Sydney, NSW, 2007, Australia

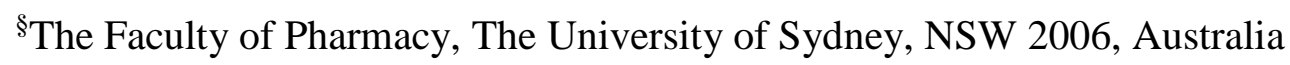

"School of Life Sciences, University of Technology Sydney, Sydney, New South Wales 2007, Australia

\section{ABSTRACT:}

Sensitivity is the key in optical detection of low-abundant analytes, such as circulating RNA or DNA. The enzyme Exonuclease III (Exo III) is a useful tool in this regard; its ability to recycle target DNA molecules results in markedly improved detection sensitivity. Lower limits of detection may be further achieved if the detection background of auto-fluorescence can be removed. Here we report an ultrasensitive and specific method to quantify trace amounts of DNA analytes in a wash-free suspension assay. In the presence of target DNA, the Exo III recycles the target DNA by selectively digesting the dye-tagged sequence-matched probe DNA strand only, so that the amount of free dye removed from the probe DNA is proportional to the number of target DNAs. Remaining intact probe DNAs are then bound onto upconversion nanoparticles (energy donor), which allows for upconversion luminescence resonance energy transfer (LRET) that can be used to quantify the difference between the free dye and tagged dye (energy acceptor). This scheme simply avoids both auto-fluorescence under infrared excitation and many tedious washing steps, as the free dye molecules are physically located away from the nanoparticle surface, and as such they remain "dark" in suspension. Compared to alternative approaches requiring enzyme-assisted amplification on the nanoparticle surface, introduction of probe DNAs onto nanoparticles only after DNA hybridization and signal amplification steps, effectively avoids steric hindrance. Via this approach, we have achieved a detection limit of 15 pM in LRET assays of human immunodeficiency viral DNA. 
2 Keywords: Exonuclease III, wash-free, suspension assay, upconversion nanoparticles, luminescence 3 resonance energy transfer.

5 Rapid detection of low-abundant specific DNA molecules becomes increasingly important to enable in6 depth studies and medical diagnosis of genetic diseases. Optical biosensors for DNA detection, including 7 colorimetric ${ }^{1}$ and fluorescent ${ }^{2-3}$ methods, have been widely used because of their high detection selectivity 8 and cost-efficiency. However, detection sensitivity is still limited due to the high background interference 9 from auto-fluorescence from the bio-samples. In addition, many fluorophores suffer serious photobleaching which leads to the poor reproducibility of the signal measurement.

12 Upconversion nanoparticles (UCNPs) $)^{4-5}$ have emerged as a new generation of optical probes for diminishing the interference of auto-fluorescence background, as they stepwise absorb two or more near-infrared 14 photons, and emit long-lifetime anti-Stokes luminescence in the visible spectrum with narrow emission bands. Each single UCNP usually contains thousands of photon sensitizers and hundreds of trivalent lanthanide ions $\left(\mathrm{Ln}^{3+}\right)$ as photon activators within an inorganic crystalline host matrix, and therefore deliver bright luminescence in the darkness via either anti-Stokes spectrum filtering or time-gated detection. Morever, distinguised from conventional organic dyes, UCNPs provide high optical stablity for better experimetal reproducibility, and also demonstrate good biocompatibility and low cytotoxicity ${ }^{6}$, as robust molecular probes for biological imaging ${ }^{7-8}$ and molecular detection. ${ }^{9-11}$

2 Using UCNPs as the donor in luminescence resonance energy transfer (LRET) systems has been broadly reported in the area of DNA detection. ${ }^{12-15}$ Replacing the organic fluorophores as classic energy donors ${ }^{16}$ by UCNPs, upconversion LRET assays are now more reliable due to low levels of auto-fluorescence background ${ }^{17}$. Moreover, due to the narrow emission bands, both the donor's and acceptor's emissions have well-defined spectrum windows with minimal levels of cross-talk for quantitative distance-dependent analysis through monitoring the decrease of the donor's intensity and increase of the acceptor's intensity. ${ }^{18-}$ ${ }^{19}$ Most of the existing DNA detection assays are based on a sandwich-type hybridization that only allows one DNA probe to detect one target DNA analyte, which holds back this technology from achieving higher detection sensitivity. 
1 Highly sensitive detection of low quantities of target DNA analytes, typically requires direct amplification 2 of the target nucleic acids by use of polymerase chain reaction (PCR $)^{20-21}$ to substantially enhance signal 3 output. However, this kind of amplification requires costly equipment, highly trained personnel, along with 4 the requirement of precise temperature control and prevention of primer dimer formation. This makes it 5 difficult to achieve widespread application of this technology. To overcome these challenges, various of 6 enzyme-free $^{22-23}$ or enzyme-assisted ${ }^{1,15,24-25}$ target-triggered signal amplification techniques, where the 7 replication of target-dependent complexes for continuous signal generation only under isothermal conditions 8 have been developed over the years. Target-triggered signal amplification assisted by Exonuclease III (Exo 9 III) enzyme for ultra-sensitive quantitation of DNA has been extensively developed and applied. ${ }^{25-28}$ Exo III has a double-strand specific, non-processive 3'-5' exo-deoxyribonulease activity that produces a step-bystep mononucleotide removal from the 3'-end of DNA. However, if the 3'-end of the DNA overhangs by more than four bases in length, it can be protected from Exo III activity. ${ }^{29}$ Typically, the mononucleotides of designed probe DNA can be stepwise removed without damaging the target DNA. This selective nucleotide digestion feature can be exploited to significantly enhance the limits of detection in DNA assays.

In this study, we introduce the Exo III-assisted target DNA-triggered signal amplification technique to further improve the detection sensitivity and simplicity of upconversion LRET for the detection and quantification of trace amounts of DNA. As illustrated in Figure 1, two in-principle schemes were designed and experimentally verified in this study. In both schemes, carboxytetramethyl rhodamine ${ }^{\mathrm{TM}}$ (TAMRA $^{\mathrm{TM}}$ ) is used as the acceptor and is conjugated to the 3 '-terminus of the single strand probe DNA. It is selected as a suitable dye due to its maximum absorption at $559 \mathrm{~nm}$, which is well matched to the green emission band of erbium doped UCNPs, resulting in its sensitized luminescence emission at $583 \mathrm{~nm}$, where conveniently UCNPs do not luminesce (Figure SI-1). The sequence of probe DNA is designed to completely hybridize with HIV target DNA. Furthermore, its 5'-end is modified with an amine group in order for it to covalently bind onto the surface of UCNPs that have been modified with polymer linkers ending in carboxyl groups. In addition, the length of the probe DNA is designed to allow the target DNA to overhang by six bases from its 3'-end. This is done to ensure the target DNA escaped digestion by Exo III, therefore allowing it to be recycled in the assay and capable of binding to remaining intact, probe DNAs (Sequences are provided in the Supporting Information). These modified probe DNAs are referred to as TAMRA-tagged probe DNAs.

Upon hybridization of target DNA to TAMRA-tagged probe DNAs (Figure 1, Scheme 1), Exo III recognizes 
the double-stranded probe-target pairs and selectively digests the TAMRA-tagged probe DNA strand, releasing free-TAMRA. Target DNA remains intact, and is capable of undertaking further rounds of hybridization to TAMRA-tagged probe DNAs. Subsequent addition of EDC-activated carboxyl-UCNPs, results in covalent attachment of the residual full length TAMRA-tagged probe DNAs - via their 5' amine group. This in turn results in LRET from the UCNPs to the bound TAMRAs by providing a proportional decrease in luminescence of the UCNPs at $539 \mathrm{~nm}$ and an increased fluorescence of the UNCP bound TAMRAs at $583 \mathrm{~nm}$ (Figure 3A). In contrast, the remaining free TAMRA dye molecules in suspension do not come into close contact with the surface of the UCNPs, contributing no effect to the LRET process. Therefore, the existence of the free TAMRAs in the solution does not influence the result of spectral measurement, eliminating time-consuming and tedious washing steps.

In the case of Scheme 2, the TAMRA-tagged probe DNAs were first conjugated onto UCNPs for efficient LRET, followed by addition of both target DNA and Exo III, designed for signal amplification. However, no obvious change was detected even in the presence of high concentrations of target DNA molecules (Figure SI-2). This comparison experiment confirms Scheme 1 as a simple, reproducible and practical distancedependent upconversion LRET assay for suspension DNA detection and quantification. In order to meet the distance requirement between UCNP and TAMRA for efficient LRET, we designed relatively short-length probe DNA sequence. As a result, in Scheme 2, short sequence DNA attached to the surface of UCNPs have caused steric hindrance effects, resulting in inhibition and/or failure of enzymatic reactions to proceed on the surface of the UCNPs.

Synthesized in organic solvent, UCNPs are hydrophobic due to their capping by surfactant ligands such as oleic acids (OA-UCNPs). Therefore subsequent surface modification to generate a hydrophilic layer is a prerequisite to yield well-dispersed nanoparticles in aqueous media for bioanalytical applications. In this work, OA-UCNPs were transferred into an aqueous environment via a ligand-exchange and carboxylfunctionalization (carboxyl-UCNPs) by POEGA-b-PMAEP, ${ }^{30}$ a copolymer composed of hydrophilic block poly(ethylene glycol) methyl ether acrylate and polymer block containing phosphate anchoring groups prepared by the versatile RAFT polymerization technique in our lab. For upconversion LRET, carbodiimide crosslinker chemistry was further applied for the conjugation of carboxyl-UCNPs with TAMRA-tagged probe DNAs (TAMRA-UCNPs) under EDC catalysis after the enzymatic digestion step. 
X-ray powder diffraction (XRD), transmission electron microscopy (TEM), dynamic light scattering (DLS),

2 Fourier transform infrared (FT-IR) spectroscopy, and X-ray photoelectron spectroscopy (XPS) were used to 3 characterize each step of the surface modification and functionalization of the UCNPs. XRD results of the 4 OA-UCNPs synthesized in this experiment corresponded with the standard pattern of the hexagonal phase $5 \quad \mathrm{NaYF}_{4}$ (Figure SI-3). TEM observations revealed that OA-UCNPs, carboxyl-UCNPs and TAMRA-UCNPs were uniform in size without obvious change in morphology (Figure 2A-C). DLS results (Figure 2D) showed that the average hydrodynamic size of UCNPs stepwise increased following the ligand-exchange surface modification and EDC coupling conjugation $(\mathrm{PDI}<0.1)$. The high-transmission electron microscopy (HRTEM) image of OA-UCNPs (Figure SI-4) shows the high crystallinity of particles with an interplanar spacing of $0.58 \mathrm{~nm}$, which is close to the $\mathrm{d}_{100}$ value of hexagonal phase $\mathrm{NaYF}_{4}{ }^{31}$ FTIR spectra were applied to identify the successful ligand exchange from OA molecules to POEGA-b-PMAEP on the surface of nanoparticles, where the peaks at $1732 \mathrm{~cm}^{-1}$ and $1103 \mathrm{~cm}^{-1}$ were respectively assigned to the stretching vibration of the carboxylic group $(\mathrm{C}=\mathrm{O})$ and phosphine oxides $(\mathrm{P}=\mathrm{O})$ in carboxyl-UCNPs. From the XPS result (Figure SI-5), the high-resolution spectra of $\mathrm{P} 2 \mathrm{p}$ (inset) further confirmed the successful modification of POEGA-b-PMAEP onto the surfaces of the UCNPs.

Figure 2F shows the luminescent spectra of OA-UCNPs in cyclohexane, carboxyl-UCNPs and ligand freeUCNPs in MES buffer, under $980 \mathrm{~nm}$ laser excitation $\left(1.36 \times 10^{6} \mathrm{~W} / \mathrm{cm}^{2}\right)$. The energy transfers from excited state to ground state have been displayed. ${ }^{32}$ Though there was no spectrum shift in the peak emission of carboxyl-UCNPs (green line), the green luminescence has been severely quenched compared with OAUCNPs (orange line). To check if the quenching was caused by the attached polymers, we removed the surface OA molecules by HCL $(0.1 \mathrm{M})$ with 20 min sonication and found that the ligand free-UCNPs (pink line) showed the same level of reduced luminescence, indicating that the quenching was possibly induced by some other factors, especially water molecules. ${ }^{33}$ The spectrum intensity changes (shown in Figure 2G) confirmed successful energy transfer from UCNPs to TAMRAs, as the fluorescence of TAMRA at $583 \mathrm{~nm}$ increased and the luminescence of UCNPs at $539 \mathrm{~nm}$ decreased. Moreover, in fact LRET can be clearly observed by the naked eye (shown as the insert photographs) with the luminescent color changing from a green (carboxyl-UCNPs, left) to yellow color (TAMRA-UCNPs, right).

In order to enhance analytical performance, the various experimental conditions were optimized (Figure 3). The molar ratio of TAMRA-tagged probe-DNAs to EDC-activated carboxyl-UCNPs is an important 
parameter for the detection sensitivity of the HIV DNA target. Increasing the amount of TAMRA-tagged probe DNAs, the fluorescence of TAMRAs at $583 \mathrm{~nm}$ was gradually enhanced with the luminescence of UCNPs at $539 \mathrm{~nm}$ reduced, and the ratiometric $\mathrm{I}_{583} / \mathrm{I}_{539}$ (shown in Figure $3 \mathrm{~A}$ ) was correspondingly increased until saturated, confirming the optimum molar ratio of TAMRA-tagged probe DNAs to UCNPs as 10:1 were used for LRET assay in this work. Meanwhile, to confirm the observation as the distance-dependent LRET rather than simple reabsorption, by mixing TAMRA-tagged probe DNAs with non EDC-activated carboxylUCNPs, though increasing the amount of TAMRA-tagged probe DNAs, no obvious change of $\mathrm{I}_{583} / \mathrm{I}_{539}$ was observed because of the long distance between TAMRA-UCNP pair (Figure 3B). The optimal reaction time of this conjugation step was also investigated and the result demonstrated that the carbodiimide chemistry between UCNPs and TAMRA-tagged probe DNAs were completely finished within one hour (Figure 3C). To ensure the complete reaction of Exo III-assisted signal amplification step is another important factor for the sensitive detection. The curve of $\mathrm{I}_{583} / \mathrm{I}_{539}$ was plotted along reaction time and shown in Figure $3 \mathrm{D}$. The reaction efficiency increased during the first forty minutes and reached steady state after one hour. The reaction time of Exo III assisted signal amplification for one hour was consequently used in this work.

For HIV detection by the Exo III-assisted upconversion LRET assay, varying concentration of target DNAs were added with fixed values of TAMRA-tagged probe DNAs (10 pmol) and Exo III (10 U) for signal amplification at $37{ }^{\circ} \mathrm{C}$ for one hour, and the solution subsequently incubated at $70{ }^{\circ} \mathrm{C}$ for 20 min to stop the digestion via deactivating the Exo III, followed by conjugation onto EDC-activated carboxyl-UCNPs for LRET at RT for one hour. Increasing the concentration of the target DNAs from $0.025 \mathrm{nM}$ to $500 \mathrm{nM}$, the fluorescent intensity of TAMRAs $(583 \mathrm{~nm})$ decreased dramatically with the luminescent intensity of UCNPs $(539 \mathrm{~nm})$ increased (Figure 4A). The formula of $\frac{\mathrm{R}_{0}\left(\frac{\mathrm{I}_{583}}{\mathrm{I}_{539}}\right)-\mathrm{R}\left(\frac{\mathrm{I}_{583}}{I_{539}}\right)}{\mathrm{R}_{0}\left(\frac{\mathrm{I}_{583}}{I_{539}}\right)}$ was applied for calculating the percentage of free dyes dropped from probe DNAs and as the Y-value of the calibration curve. R refers to experimental group in the presence of target and $\mathrm{R}_{0}$ refers to control group in the absence of target with Exo III-assisted signal amplification. The changing curve of $\left(\mathrm{R}_{0}-\mathrm{R}\right) / \mathrm{R}_{0}$ versus the incremental concentrations of target DNAs was plotted as Figure 4B. The trend of the reaction followed a sigmoid increase and the curve was perfectly fitted with Growth/Sigmoidal-Hill 1 equation $\left(r^{2}=0.99\right)$. Limit of detection (LOD) via stepwise diluting the concentration of target DNAs at signal amplification step, was determined by the value of $\overline{\mathrm{x}}+3 \mathrm{SD},{ }^{34}$ where $\overline{\mathrm{x}}$ is the mean value and SD is the standard deviation among three trials of experiment. The reaction in the absence of target DNAs was considered as blank $(\mathrm{n}=5)$. The experimental LOD value in this work is $15 \mathrm{pM}$, 
1 equal to $0.3 \mathrm{fmol}$, with relative standard deviation (RSD) within $15 \%$.

2

3 To further evaluate the specificity of the as-developed upcoversion LRET assay, TAMRA-tagged probe 4 DNAs and Exo III were incubated with 1-base/3-base mis-match, non-match DNA1 (random sequence) and 5 non-match DNA2 (HBV sequence), respectively, and with three replicate tests for each group. The results 6 displayed in Figure 5, showed that this assay method can differentiate between all four mis-/non-match 7 groups with HIV target group ( $\mathrm{p}<0.05)$, suggesting its potential for single-nucleotide polymorphisms (SNPs) 8 assays.

9

10 In summary, we have developed an ultra-sensitive and specific detection and quantification method for trace

11 amounts of DNA via an Exo III-assisted target DNA-triggered signal amplification technique and 12 upconversion LRET assay. The Exo III can work under isothermal condition without costly and 13 sophisticated equipment. The distance-dependent energy transfer avoids the influence of the unbound 14 TAMRA molecules eliminating additional time-consuming washing step and providing a quicker test result 15 turnout. The experimental LOD value in this work is $15 \mathrm{pM}$, one order of magnitude enhanced sensitivity of 16 DNA detection in comparison to the conventional DNA-DNA direct hybridization upconversion LRET 17 assay. ${ }^{12-15}$ This wash-free suspension DNA assay has numerous potential clinical applications, along with its 18 main features of high sensitivity, rapid speed, simplicity and low cost. 


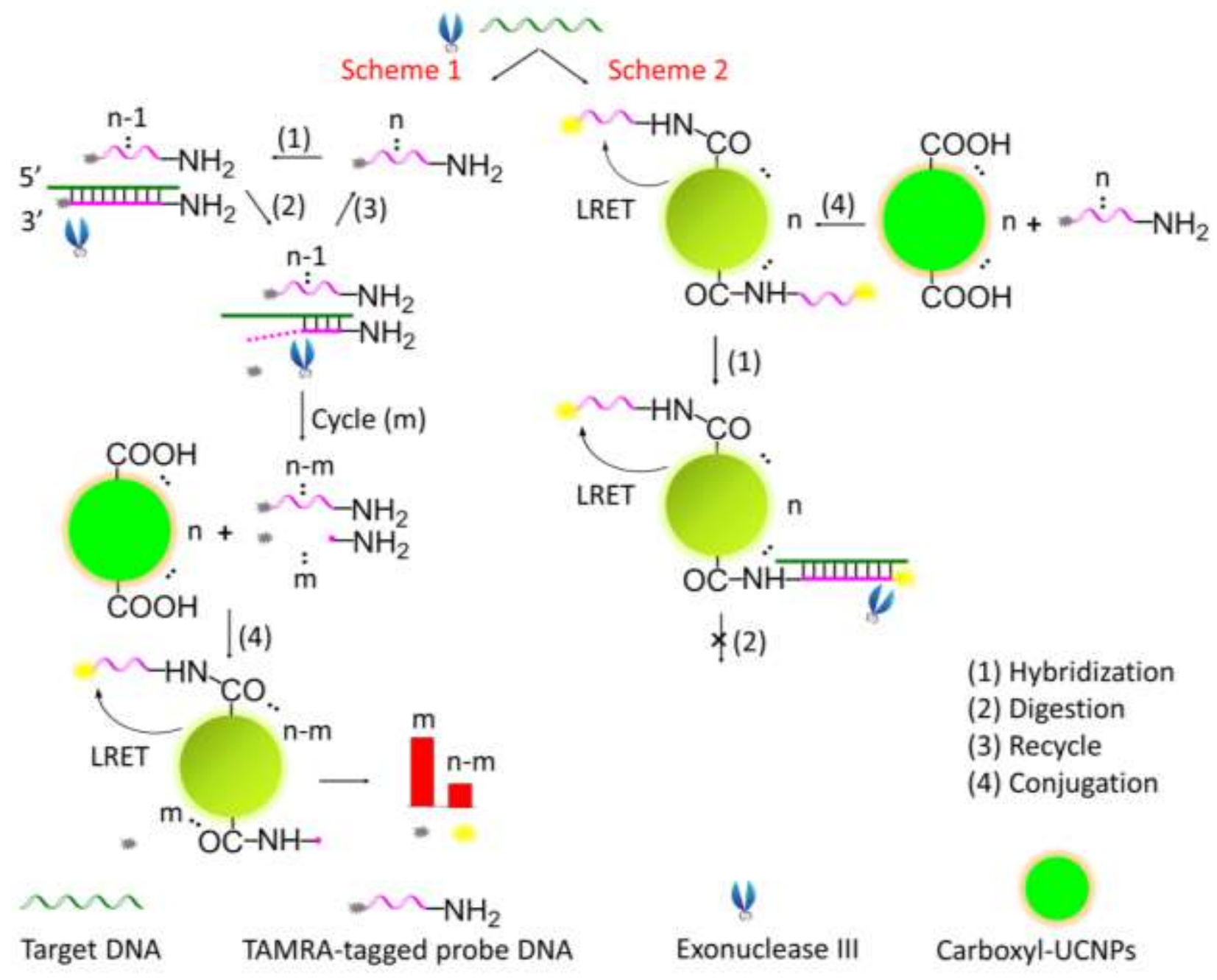

Figure 1. Schemas $1 \& 2$, illustrating the use of Exonuclease III-assisted upconversion resonance energy transfer in a wash-free suspension DNA assay. 

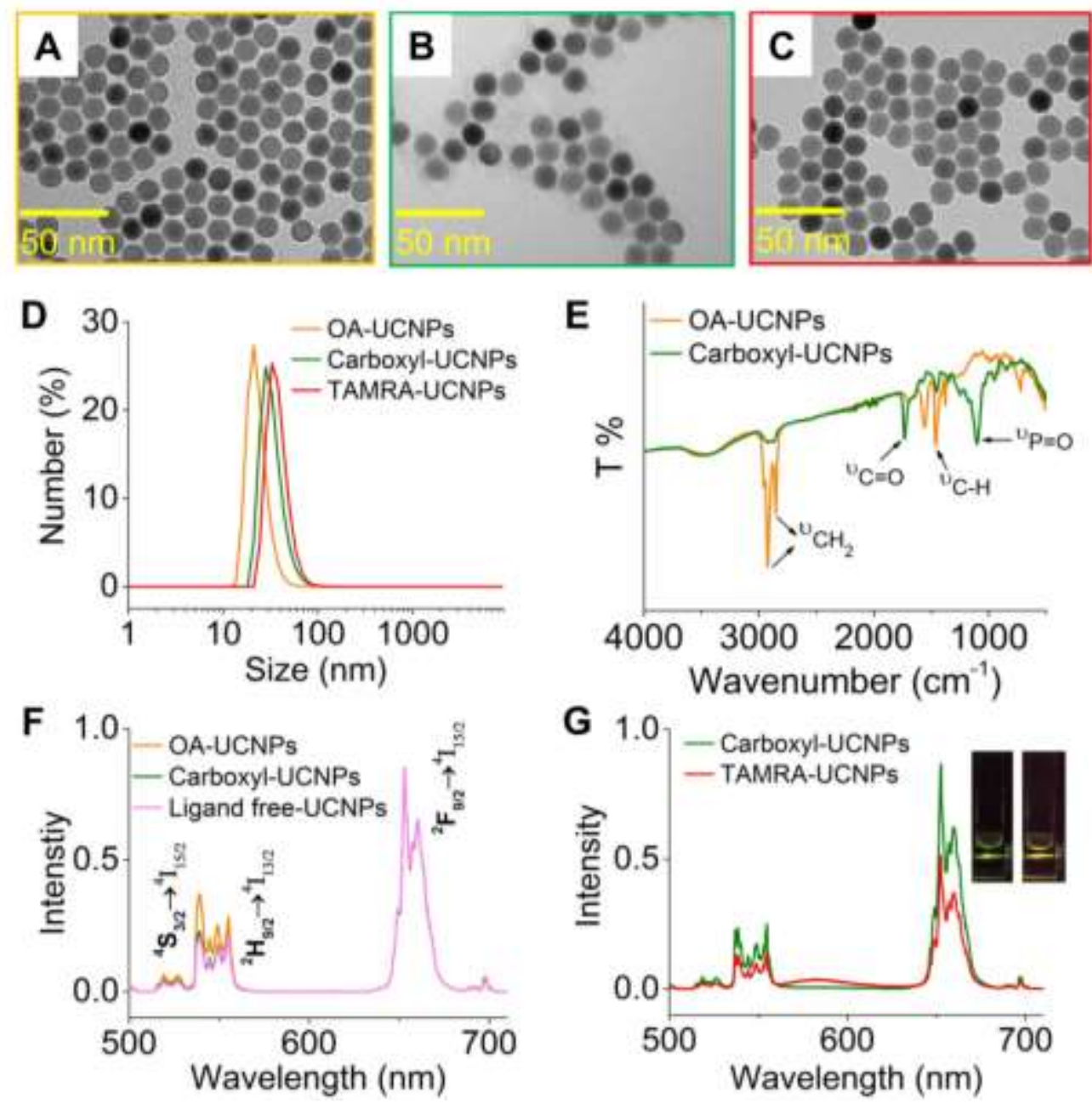

Figure 2. TEM images of (A) OA-UCNPs, (B) carboxyl-UCNPs and (C) TAMRA-UCNPs. (D) Size distribution histogram of OA-UCNPs, carboxyl-UCNPs and TAMRA-UCNPs. (E) FT-IR spectra of OAUCNPs and carboxyl-UCNPs. (F) Luminescence spectra of OA-UCNPs, carboxyl-UCNPs and ligand freeUCNPs. (G) Luminescence spectra of carboxyl-UCNPs and TAMRA-UCNPs. The inset shows photographs of (left) carboxyl-UCNPs and (right) TAMRA-UCNPs under excitation at $980 \mathrm{~nm}$. 

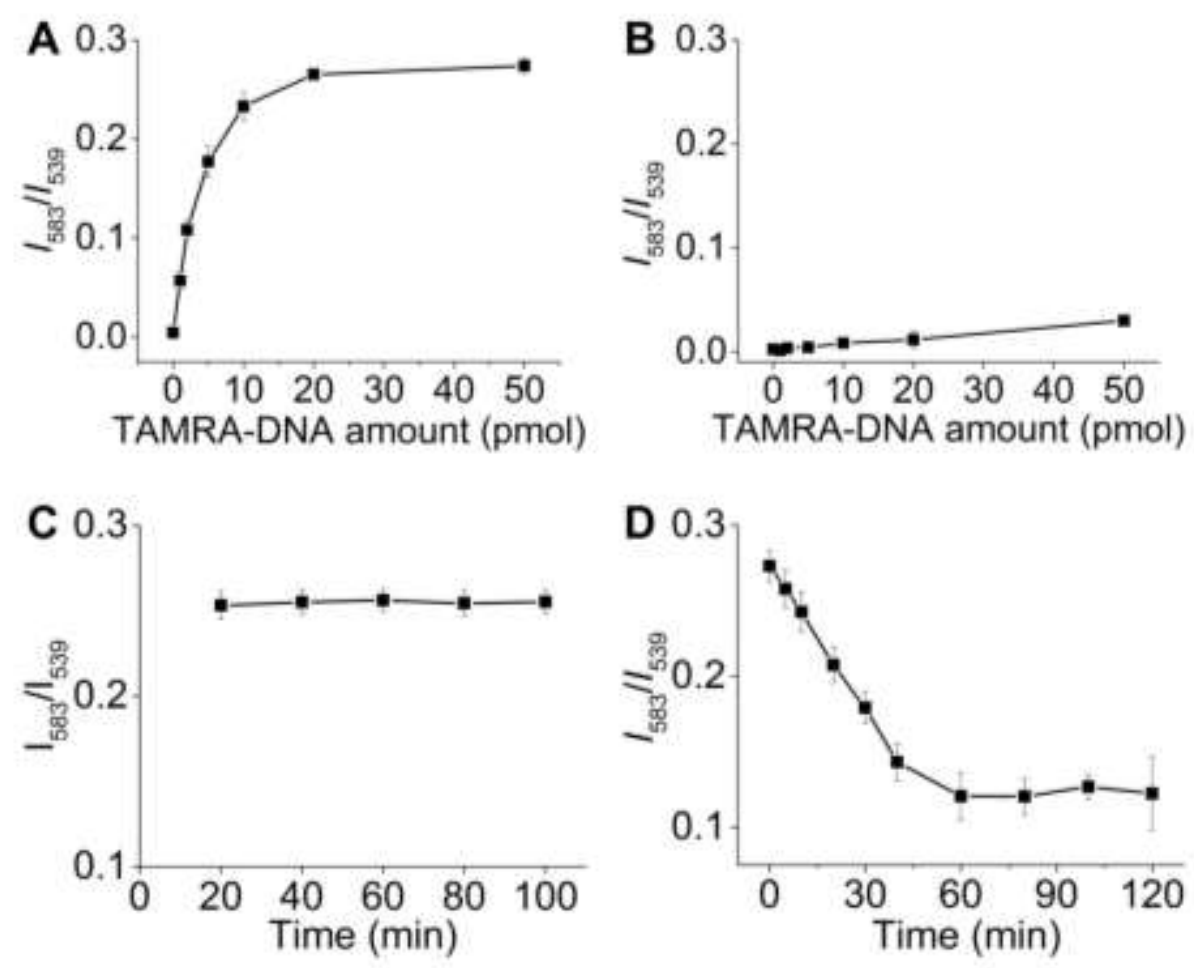

Figure 3. Optimization of Exonuclease III-assisted upconversion resonance energy transfer in wash-free suspension DNA assay. (A) $\mathrm{I}_{583} / \mathrm{I}_{539}$ along with incremental amounts of TAMRA-tagged probe DNAs (1, 2, 5, 10, 20 and 50 pmol) with EDC catalysis. (B) $\mathrm{I}_{583} / \mathrm{I}_{539}$ along with incremental amounts of TAMRA-tagged probe DNAs (1, 2, 5, 10, 20 and 50 pmol) without EDC catalysis. (C) $\mathrm{I}_{583} / \mathrm{I}_{539}$ along with the reaction time for carboxyl-UCNPs conjugation with TAMRA-tagged probe DNAs catalyzed by EDC at different time points $(20,40,60,80$ and $100 \mathrm{mins})$. (D) $\mathrm{I}_{583} / \mathrm{I}_{539}$ along with the reaction time $(0,5,10,20,30,40,60,80$, 100 and 120 mins) at Exo III digestion step in the presence of $10 \mathrm{nM}$ target DNAs. For each figure, the error bars represent the standard deviation calculated from three parallel groups. 

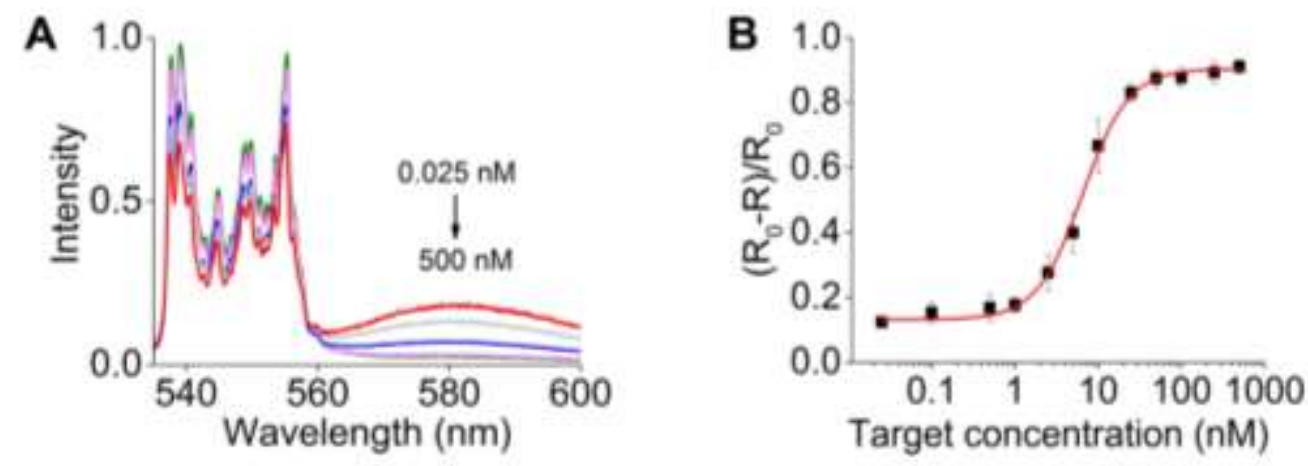

Figure 4. Detection sensitivity in Exonuclease III-assisted upconversion resonance energy transfer in a wash-free suspension DNA assay. (A) Luminescence spectra of Exo III-assisted upconversion LRET DNA assay upon incremental concentrations of target DNAs. (B) The calibration curve along with various concentrations of target DNAs $(0.025,0.1,0.5,1,2.5,5,10,25,50,100,250$ and $500 \mathrm{nM})$. Hill 1 model with 4-parameter equation was applied for curve fitting. The error bars represent the standard deviation calculated from five parallel groups. 


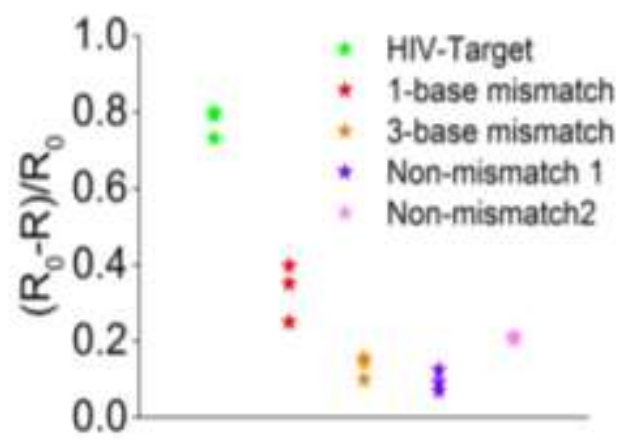

Figure 5. Specificity of the Exonuclease III-assisted upconversion resonance energy transfer in a wash-free suspension DNA assay. Scatter plot shows the $\left(\mathrm{R}-\mathrm{R}_{0}\right) / \mathrm{R}_{0}$ value for each group respectively. 


\section{REFERENCES}

(1) Xu, W.; Xue, X.; Li, T.; Zeng, H.; Liu, X. Angew. Chem. Int. Ed. 2009, 48 (37), 6849-6852.

(2) Smith, L. M.; Sanders, J. Z.; Kaiser, R. J.; Hughes, P.; Dodd, C.; Connell, C. R.; Heiner, C.; Kent, S.; Hood, L. E. Nature 1985, 321 (6071), 674-679.

(3) Ren, W.; Liu, H.; Yang, W.; Fan, Y.; Yang, L.; Wang, Y.; Liu, C.; Li, Z., Biosens. Bioelectron. 2013, 49, 380-386.

(4) Wu, S.; Han, G.; Milliron, D. J.; Aloni, S.; Altoe, V.; Talapin, D. V.; Cohen, B. E.; Schuck, P. J. Proc. Natl. Acad. Sci. 2009, 106 (27), 10917-10921.

(5) Wang, F.; Han, Y.; Lim, C. S.; Lu, Y.; Wang, J.; Xu, J.; Chen, H.; Zhang, C.; Hong, M.; Liu, X. Nature 2010, 463 (7284), 1061-1065.

(6) Chatterjee, D. K.; Rufaihah, A. J.; Zhang, Y. Biomaterials 2008, 29 (7), 937-943.

(7) Nyk, M.; Kumar, R.; Ohulchanskyy, T. Y.; Bergey, E. J.; Prasad, P. N. Nano lett. 2008, 8 (11), 3834-3838.

(8) Wang, J.; Wei, T.; Li, X.; Zhang, B.; Wang, J.; Huang, C.; Yuan, Q. Angew. Chem. Int. Ed. 2014, 126 (6), 1642-1646.

(9) Deng, R.; Xie, X.; Vendrell, M.; Chang, Y.-T.; Liu, X. J. Am. Chem. Soc. 2011, 133 (50), 20168-20171.

(10) Van de Rijke, F.; Zijlmans, H.; Li, S.; Vail, T.; Raap, A. K.; Niedbala, R. S.; Tanke, H. J. Nat. Biotechnol. 2001, 19 (3), 273-276.

(11) Wang, L.; Yan, R.; Huo, Z.; Wang, L.; Zeng, J.; Bao, J.; Wang, X.; Peng, Q.; Li, Y. Angew. Chem. Int. Ed. 2005, 44 (37), 6054-6057.

(12) Zhang, P.; Rogelj, S.; Nguyen, K.; Wheeler, D. J. Am. Chem. Soc. 2006, 128 (38), 12410-12411.

(13) Liu, J.; Cheng, J.; Zhang, Y. Biosens. Bioelectron. 2013, 43, 252-256.

(14) Zhu, H.; Lu, F.; Wu, X. C.; Zhu, J. J. Analyst 2015, 140 (22), 7622-7628.

(15) Wang, P.; Ahmadov, T. O.; Lee, C.; Zhang, P. RSC Adv 2013, 3 (37), 16326-16329.

(16) Sapsford, K. E.; Berti, L.; Medintz, I. L. Angew. Chem. Int. Ed. 2006, 45 (28), 4562-4589.

(17) Su, Q.; Feng, W.; Yang, D.; Li, F., Acc. Chem. Res. 2016, 50(1), 32-40.

(18) Deng, R.; Wang, J.; Chen, R.; Huang, W.; Liu, X. J. Am. Chem. Soc. 2016, 138 (49), 15972-15979.

(19) Soukka, T.; Rantanen, T.; Kuningas, K. Ann. N. Y. Acad. Sci. 2008, 1130 (1), 188-200.

(20) Heim, A.; Ebnet, C.; Harste, G.; Pring-Åkerblom, P. J. Med. Virol. 2003, 70 (2), 228-239.

(21) Heid, C. A.; Stevens, J.; Livak, K. J.; Williams, P. M. Genome Res. 1996, 6 (10), 986-994.

(22) Gao, F.; Lei, J.; Ju, H. Anal. Chem. 2013, 85 (24), 11788-11793.

(23) Gao, F.; Fan, T.; Wu, J.; Liu, S.; Du, Y.; Yao, Y.; Zhou, F.; Zhang, Y.; Liao, X.; Geng, D. Biosens. Bioelectron 2017, 96, 62-67.

(24) Bi, S.; Zhang, J.; Zhang, S. Chem. Comm. 2010, 46 (30), 5509-5511.

(25) Gao, F.; Lei, J.; Ju, H. Chem. Comm. 2013, 49 (68), 7561-7563.

(26) Zuo, X.; Xia, F.; Xiao, Y.; Plaxco, K. W. J. Am. Chem. Soc. 2010, 132 (6), 1816-1818.

(27) Xuan, F.; Luo, X.; Hsing, I. M. Anal. Chem. 2012, 84 (12), 5216-5220.

(28) Lu, J.; Paulsen, I. T.; Jin, D. Anal. Chem. 2013, 85 (17), 8240-8245.

(29) Weiss, B. J. Biol. Chem. 1976, 251 (7), 1896-1901.

(30) Duong, H. T. T.; Chen, Y.; Abbas, S.; Wen, S.; Parviz, M.; Ford, M.; Shimoni, O.; Jin, D., 'Systematic Investigation of Functional Ligands for Colloidal Stable Upconversion Nanoparticles ' Submitted.

(31) Liu, D.; Xu, X.; Du, Y.; Qin, X.; Zhang, Y.; Ma, C.; Wen, S.; Ren, W.; Goldys, E. M.; Piper, J. A. Nat. Commun. 2016, 7, 10254.

(32) Zhou, J.; Gu, F.; Liu, X.; Qiu, J. Adv. Opt. Mater. 2016, 4 (8), 1174-1178.

(33) Arppe, R.; Hyppänen, I.; Perälä, N.; Peltomaa, R.; Kaiser, M.; Würth, C.; Christ, S.; Resch-Genger, U.; Schäferling, M.; Soukka, T. Nanoscale 2015, 7 (27), 11746-11757.

(34) Armbruster, D. A.; Tillman, M. D.; Hubbs, L. M. Clin. Chem. 1994, 40 (7), 1233-1238. 


\section{Acknowledgments}

The authors acknowledge the financial support from the Australian Research Council (ARC) Future Fellowship Scheme (D.J., FF130100517), National Health and Medical Research Council (NHMRC) Dementia Research Fellowship (O.S., APP1101258) and ARC Industry Transformational Research Hub Scheme (D.J., O.S., S.V., IH150100028). XPS measurements were undertaken on the Soft X-ray Spectroscopy beamline at the Australian Synchrotron, and the technical assistance of Dr Anton Tadich and Mr Christian C. Clarke are highly appreciated. 


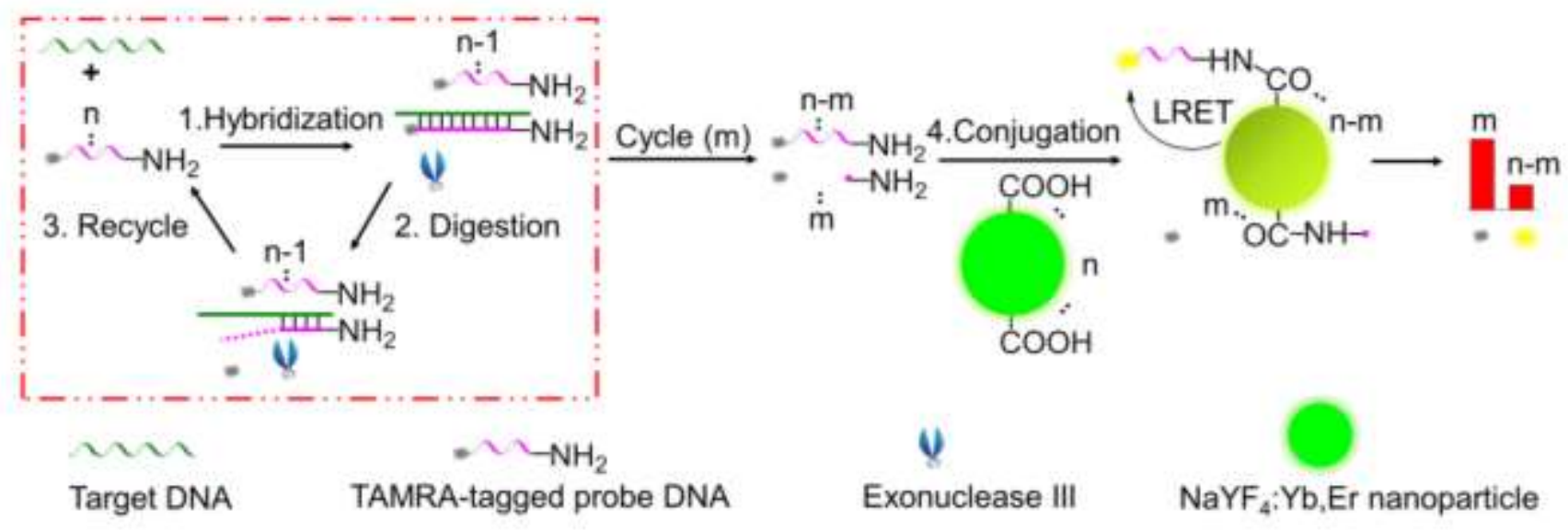

For Table of Contents Only 